\title{
Successful Genetic Transformation of Modern and Ancient Wheats (Triticum L.) spp.: Effect of Tissue Culture and Germplasm Ploidy
}

\author{
Dmitry Miroshnichenko ${ }^{1,2}$, Tatiana Savchenko ${ }^{1}$, Alexander Pushin ${ }^{1,2}$, Sergey Dolgov ${ }^{2}$ \\ ${ }^{1}$ Institute of Basic Biological Problems RAS, \\ 142290, Pushchino, Russian Federation \\ miroshnichenko@bibch.ru \\ ${ }^{2}$ Branch of Shemyakin and Ovchinnikov Institute of Bioorganic Chemistry RAS \\ 142290, Pushchino, Russian Federation
}

\section{Extended Abstract}

Wheat is one of the most important cereal crops in the world. Modern biotechnologies such as genetic transformation, gene targeting and genome editing are becoming powerful research tools in the process of cereal improvement. In recent years there has been significant progress in tissue culture and genetic transformation in bread wheat (T. aestivum L.) and durum wheat (T. durum L.). For other wheat species no significant progress has been made. Here we present a routine protocol for production of fertile transgenic plants of di-, tetra- and hexaploid wheat species.

The genetic transformation of polyploid wheats requires the adjustment of the gene transfer conditions considering the significant germplasm impact on the tissue culture efficiency. In our experiments we firstly analyzed various germplasms for the ability to develop morphogenic structures in vitro. The conventional protocol, that included 4-week cultivation of isolated immature embryos under darkness on the media containing 2,4-D was used. Experiments revealed that tissue cultures of tetraploid wheats with AABB genome demonstrated the better morphogenic abilities and efficiency of plant regeneration (up to 100\%) rather than the wheats with other genomic formulas. Low morphogenic response (0-15\%) was demonstrated by diploid einkorn (AA genome) and wheat with G genome, especially tetraploid T.timopheevii (AAGG genome). For this reason various types of plant growth regulators, sugars and organic compounds, their concentration and combination were studied to achieve satisfactory morphogenic response (up to 90\%) in recalcitrant wheat species prior to the adjustment of the gene transfer conditions.

The optimization of parameters for the transfer of heterologous sequences into the cells of polyploid wheats was performed by means of particle inflow gun using the combination of gfp as vital reporter gene and bar genes for transgene resistance. Such dual selection strategy allowed establishing the efficient escape-free transformation protocols for einkorn, emmer wheat (AABB genome), timopheevii wheat and bread wheat (AABBDD genome). Plants derived from independent transformation events were reproducibly regenerated in a relatively short time (3-5 month), and the integrated transgenes are successfully inherited and expressed by the progeny. Despite the fact that only highly morphogenic germplasms were used for the transfer of genes, the species-dependant influence on the transformation efficiency was still observed. Tetraploid emmer wheat demonstrated very high potential for genetic transformation (in average 13.0\%), while diploid einkorn and tetraploid timopheevii wheat were still recalcitrant $(0.5 \%)$; modern hexaploid bread wheat varieties displayed moderate gene transfer efficiency (1.3-2.8\%). In any case transgenic einkorn and timopheevii wheat as far as we know have been generated for the first time.

The developed protocol was further tested to produce polyploid wheats with the altered content of jasmonates by expression of Arabidopsis gene AtOPR3 (12-oxophytodienoate reductase 3). As before, the number of transgenic plants that formed after the bombardment of emmer wheat was three times greater than the same of hexaploid bread wheat.

We believe that proposed protocol opened the way to easily produce transgenic wheats for various biotechnology projects including both applied aspects and basic research.

The work was supported by the Russian Science Foundation, grant No 16-14-10155. 